\title{
The role of osteoblasts in regulating hematopoietic stem cell activity and tumor metastasis
}

\section{K. Neiva ${ }^{1}$, \\ Y.-X. Sun ${ }^{2}$ and \\ R.S. Taichman ${ }^{2}$}

${ }^{1}$ Program in Oral Health Sciences, ${ }^{2}$ Department of Periodontics, Prevention,
Geriatrics, School of Dentistry, University of Michigan, Ann Arbor, MI, USA

\section{Correspondence \\ R.S. Taichman \\ Department of Periodontics \\ Prevention, Geriatrics \\ School of Dentistry \\ University of Michigan \\ 1011 North University Ave. \\ Ann Arbor, MI 48109-1078 \\ USA \\ E-mail: rtaich@umich.edu}

Presented at SIMEC 2004

(International Symposium

on Extracellular Matrix),

Angra dos Reis, RJ, Brazil,

September 27-30, 2004.

Research supported in part by the Tissue Procurement Core of the University of Michigan Comprehensive Cancer Center, Grant \#CA46952, and directly supported by developmental grants from the University of Michigan Core Centers for Musculoskeletal Disorders (AR46024) and awards from the National Institutes of Health (DE13701, PO1-CA-93900). ...................

Received February 16, 2005 Accepted April 12, 2005

\begin{abstract}
Bone marrow stromal cells are critical regulators of hematopoiesis. Osteoblasts are part of the stromal cell support system in bone marrow and may be derived from a common precursor. Several studies suggested that osteoblasts regulate hematopoiesis, yet the entire mechanism is not understood. It is clear, however, that both hematopoietic precursors and osteoblasts interact for the production of osteoclasts and the activation of resorption. We observed that hematopoietic stem cells (HSCs) regulate osteoblastic secretion of various growth factors, and that osteoblasts express some soluble factors exclusively in the presence of HSCs. Osteoblasts and hematopoietic cells are closely associated with each other in the bone marrow, suggesting a reciprocal relationship between them to develop the HSC niche. One critical component regulating the niche is stromal-derived factor-1 (SDF-1) and its receptor CXCR4 which regulates stem cell homing and, as we have recently demonstrated, plays a crucial role in facilitating those tumors which metastasize to bone. Osteoblasts produce abundant amounts of SDF-1 and therefore osteoblasts play an important role in metastasis. These findings are discussed in the context of the role of osteoblasts in marrow function in health and disease.
\end{abstract}

Key words

- Osteoblasts

- Hematopoietic stem cells

- Bone marrow

- Stromal-derived factor-1

- CXCR4

- Prostate cancer

\section{Osteoblast-hematopoietic stem cell interactions}

In adults, the bone marrow is the sole microenvironment that supports all activities of normal hematopoiesis. Hematopoietic stem cells (HSCs) home to the bone marrow during development and transplantation, where maintenance of pluripotency and maturation is facilitated (1). Both of these activities require different growth fac- tors and cellular contexts. Bone marrow stromal cells are comprised of different cellular populations including fibroblasts, endothelial and reticular cells, adipocytes and osteoblasts which serve as a rich source of growth factors. At present, it is difficult to discern the relative importance of each of these populations in hematopoiesis. However, most evidence points to an important functional dialogue between HSCs and bone stromal cells $(2,3)$. HSCs and stromal cells collaborate to 
produce the extracellular bone marrow matrix, in which cytokine synthesis results in the formation of various blood cells (4-6).

In the marrow, HSC are in close proximity to endosteal bone surfaces rather than being randomly distributed throughout the marrow cavity $(7,8)$. These observations and the recent demonstrations that HSCs lodge near endosteal surfaces during bone marrow engraftment $(9,10)$ suggest that the resident cells are critical regulators of stem cell trafficking. Mature osteoblasts are found on endosteal surfaces and share several phenotypic characteristics with the hematopoieticsupportive stromal cell lines $(1,11-13)$. Osteoblasts are cells of mesenchymal origin that are primarily responsible for the formation of a calcified extracellular matrix. In mammals, osteoblast-derived matrices serve several essential functions: support for locomotion, reservoir for essential minerals, and housing to protect vital organs from injury.

Previous investigations have shown that in order to survive, in the absence of exogenous cytokines, hematopoietic progenitors and long-term culture-initiating cells (LTCIC) must be co-cultured with stromal cells (14). We have observed that osteoblasts can support progenitor and LTC-IC activity $(12,15,16)$ and facilitate engraftment across HLA boundaries (17). Moreover, we have demonstrated that proximity of $\mathrm{CD} 34^{+}$bone marrow cells to osteoblasts induces the synthesis of several cytokines by osteoblasts $(6,18)$. However, progenitor cells and LTCIC survival on osteoblasts requires direct cell-cell contact, despite the elaboration of soluble cytokines, which are necessary but not sufficient for the maintenance of hematopoietic cells on osteoblasts. This suggests that direct cell-cell contact of HSCs on osteoblasts is critical to ensure HSC survival.

We have also observed that HSCs regulate osteoblastic secretion of IL-6, MIP- $1 \alpha$, and other factors, possibly directing the formation of a suitable microenvironment for
HSC engraftment (19). Osteoblasts also express soluble factors that cannot be detected in the absence of HSCs, again possibly to keep HSCs quiescent (19). The data strongly suggest that osteoblasts are intimately involved in regulating hematopoietic cell proliferation and survival in vitro. The fact that osteoblasts and hematopoietic cells are closely associated with each other in the bone marrow indicates that osteoblasts may modulate normal hematopoiesis in vivo. These findings strongly suggest a reciprocal relationship between osteoblasts and hematopoietic cells, but the dimension of these interactions has yet to be defined.

Recently, several groups reported the identification of a hematopoietic stem cell niche (20-22). These reports suggested that osteoblasts are critical regulators of hematopoiesis (23). Zhang et al. (20), using mice with a conditional inactivation of bone morphogenic protein (BMP) receptor type IA demonstrated that nearly twice the number of HSCs relative to wild-type mice were present in the marrow. Co-localization analyses or the distribution of the $\mathrm{N}$-cadherin ${ }^{+}$ osteoblastic cells revealed a similar distribution pattern of HSCs, where $\mathrm{N}$-cadherin was asymmetrically localized to the cell surface of HSCs. By over-expressing a constitutively active parathyroid hormone receptor, Calvi et al. (21) observed an increased number of HSCs in the marrow. In this case, Notch-1 signaling in HSCs matched closely with Jagged-1 receptors on osteoblasts. Moreover, treatment of normal animals with an anabolic regime of parathyroid hormone similarly expanded the HSC population in the marrows, possibly due to an expansion of osteoblast precursors. A third group implicated Tie2 in the development of the stem cell niche. This study built on the finding that Tie 1 and Tie 2 receptors are not required for fetal hematopoiesis nor for the emergence of definitive HSCs but are required to home to the bone marrow (24). Arai et al. (22) demonstrated that Ang-1 expressed by osteo- 
blasts activates Tie 2 on the stem cells and promotes tight adhesion of stem cells to their niche. Presumably this adhesion results in HSC quiescence and survival, with consequent stem cells' maintenance and self-renewal.

Other studies suggest a role for osteoblasts in immunoregulation and HSC transplantation. Lineage-depleted hematopoietic progenitor cells may engraft within, but not across the MHC antigen barriers. El-Badri et al. (17) demonstrated that using osteoblasts in a transplant graft may facilitate HSC engraftment between mouse strains. Transplanted mice were free of disease and fully reconstituted. Thus, osteoblasts may represent an essential component of the stromal cell population that facilitates engraftment of marrow stem cells in an allogenic environment. Significant success in the ability to replace the bone marrow stromal cell component in 5 patients suffering from different life-threatening diseases was recently demonstrated. A heterogeneous transplantation approach was used, comprised of transplanting bone fragments placed both intraperitoneally and directly into bone along with cultured osteoblast-like cells and fully matched marrow grafts $(25,26)$. This method of using transplantation of bone fragments and cultured stromal cells from bone has made possible successful engraftment of donor stem cell/stromal cells. Yet how osteoblasts facilitate these events is not clear. Older data show that osteoblasts may present antigens (27) and, based upon their phenotype, may even function as dendritic cells (28). Clearly understanding their role in transplantation is becoming increasingly important for a number of clinical settings including HSC transplantation.

\section{Stromal-derived factor-1 (SDF-1 or CXCL12) and CXCR4 in tumor metastasis}

One protein that is likely to be particu- larly important in osteoblast-HSC interactions is SDF-1 or CXCL12. The chemokine SDF-1 and its receptor CXCR4 play a role in B-cell lymphopoiesis and myelopoiesis and SDF-1 acts as a chemotactic factor for Tcells and monocytes. SDF-1 is produced by bone marrow stromal cells also in the absence of stimuli generated by viral or bacterial infections, suggesting a major role for SDF-1 in steady-state homeostatic processes such as the control of leukocyte trafficking and retention of hematopoietic stem and progenitor cells within the bone marrow. SDF1 has also been identified as a powerful chemoattractant for immature and mature hematopoietic cells of several lineages (2931). Gene deletion of SDF-1 or CXCR4 results in normal fetal liver hematopoiesis, but marrow engraftment by hematopoietic cells is not observed (32-34). In addition, overexpression of CXCR4 by human hematopoietic progenitor cells and other blood cells enhances, whereas anti-CXCR4 antibodies inhibit, marrow engraftment in nude mice (34).

Typical of molecules that regulate cell trafficking, SDF-1 and its receptor have dynamic and complementary expression patterns during organogenesis (35). We have recently demonstrated that osteoblasts and marrow endothelial cells express SDF-1 to localize human hematopoietic progenitor cells into the marrow $(34,36,37)$. In the bone marrow SDF-1 is constitutively produced by osteoblasts, fibroblasts and endothelial cells. Osteoblast-produced SDF-1 may be a mechanism for the selective attraction of circulating osteoclast precursors into bone and their migration within marrow to appropriate perivascular stromal sites for RANKL differentiation into restorative osteoclasts $(38,39)$. Since the formation of the marrow required the coordinated action of osteoblasts and osteoclastic precursors, the absence of osteoblasts producing SDF-1 or hematopoietic cells expressing CXCR4 could limit osteoclastic and subsequent HSC recruitment into the marrow cavity. Thus, SDF- 
1 and CXCR4 represent at least one of the critical determinants for bone marrow homing by hematopoietic cells.

Many cancers have a predisposition to metastasize to bone, including prostate and breast cancer, resulting in significant morbidity and mortality. However, what defines the molecular and cellular predilection for these tumors to metastasize to bone is not understood. Multiple factors are involved in the development of tumor in bone marrow, including the acquisition of metastatic abilities by the cancer cell, chemotactic responses to bone-derived factors, preferential adhesion to bone marrow endothelium, and the interaction of cancer cells with the bone microenvironment.

One prerequisite for molecules involved in the metastasis of tumor cells is their expression in endothelial cells of the target organ, since before invasion tumor cells must first attach to the endothelial cells of vessels in specific organs. SDF-1 expression has been confirmed in endothelial cells of human arterioles and arteries from lung, liver and bone marrow (40). Moreover, SDF-1 may have autocrine-like effects to promote matrix-dependent endothelial tube formation and vascularization during organogenesis $(41,42)$. These results suggest that the SDF-1/CXCR4 axis plays a role in angiogenesis, and the endothelial-derived SDF-1 functions as a stimulating agent for tumor cells that express the CXCR4 receptor.

We have demonstrated that the SDF-1/ CXCR4 chemokine axis is activated in prostate cancer $(\mathrm{CaP})$ metastasis to bone (19). Specifically, CXCR4 expression is related to increasing $\mathrm{CaP}$ grade (43). Moreover, we have demonstrated that SDF-1 signaling through CXCR4 triggers the adhesion of $\mathrm{CaPs}$ to bone marrow endothelial cells. Similar demonstrations have also been made suggesting that the SDF-1/CXCR4 axis may play parallel roles in other tumors that also metastasize to the marrow (44). Furthermore, antibody to CXCR4 blocks the meta- static spread of the tumors to the lung and lymph nodes (45). Thus, for the last several years our laboratory has focused on delineating the role of SDF-1/CXCR4 receptor in $\mathrm{CaP}$ disease. Recently, we performed two independent studies. First, to establish a positive correlation between SDF-1 gene and protein expression with tumor metastasis, SDF-1 levels were characterized for a variety of murine tissues by ELISA and in situ hybridization (45). The role of CXCR4 in metastasis of $\mathrm{CaP}$ to bone was examined in an in vivo metastasis model. In this model, neutralizing antibody to CXCR4 limited the extent of bone metastases. Finally, antibody and blocking peptide to CXCR4 limited the growth of intraosseous CaPs following $\mathrm{CaP}$ cell intratibial injections (45). Taken together, these in vivo metastasis data provide critical support for a role of SDF-1/CXCR4 in skeletal metastasis. Most importantly, these novel data demonstrate that SDF-1/CXCR4 participates in localizing tumors to the bone marrow in prostate cancer and may help in the design of therapeutic treatments to prevent the spread and growth of metastasis.

Despite the significant voids in our knowledge, there are good reasons to suspect that osteoblast-derived factors play a central role in hematopoietic development in vivo and now in tumor metastasis. In the marrow, osteoblasts are in a biologically relevant site to transmit information to the developing hematopoietic lineages. Moreover, osteoblasts produce factors that influence blood cell development. Identification of osteoblast function regarding hematopoiesis, being they restrictive and/or stimulatory, will yield significant insights into the functional relationships of this complex tissue. These findings may be useful to re-engineer the marrow organ in patients with myeloproliferative disorders. By further exploring the SDF-1/CXCR4 axis, we believe that new therapeutic strategies will emerge to block tumor metastasis and to modulate the trafficking of hematopoietic cells. 


\section{References}

1. Nelissen JM, Torensma R, Pluyter M et al. (2000). Molecular analysis of the hematopoiesis supporting osteoblastic cell line U2-OS. Experimental Hematology, 28: 422-432.

2. Satoh $\mathrm{M}$, Mioh $\mathrm{H}$, Konishi $\mathrm{N}$ et al. (1997). Characterization of the molecules involved in the hematopoietic microenvironment provided by mouse stromal cell line MC3T3-G2/PA6 using a unique reporter system that analyzes the direct cell-to-cell interaction. Acta Haematologica, 98: 95-103.

3. Verfaillie CM (1992). Direct contact between human primitive hematopoietic progenitors and bone marrow stroma is not required for long-term in vitro hematopoiesis. Blood, 79: 2821-2826.

4. Gupta P, Blazar BR, Gupta K et al. (1998). Human CD34(+) bone marrow cells regulate stromal production of interleukin- 6 and granulocyte colony-stimulating factor and increase the colony-stimulating activity of stroma. Blood, 91: 3724-3733.

5. Koller MR, Oxender M, Jensen TC et al. (1999). Direct contact between CD34(+)lin(-) cells and stroma induces a soluble activity that specifically increases primitive hematopoietic cell production. Experimental Hematology, 27: 734-741.

6. Taichman RS, Reilly MJ, Verma RS et al. (1997). Augmented production of interleukin- 6 by normal human osteoblasts in response to CD34+ hematopoietic bone marrow cells in vitro. Blood, 89: 11651172.

7. Gong J (1978). Endosteal marrow: a rich source of hematopoietic stem cells. Science, 199: 1443-1445.

8. Islam A, Glomski C \& Henderson ES (1990). Bone lining (endosteal) cells and hematopoiesis: a light microscopic study of normal and pathologic human bone marrow in plastic-embedded sections. Anatomical Record, 227: 300-306.

9. Nilsson SK, Dooner MS, Tiarks CY et al. (1997). Potential and distribution of transplanted hematopoietic stem cells in a nonablated mouse model. Blood, 89: 4013-4020.

10. Quesenberry PJ \& Becker PS (1998). Stem cell homing: rolling, crawling, and nesting [comment]. [Review] [54 refs]. Proceedings of the National Academy of Sciences, USA, 95: 15155-15157.

11. Dorheim MA, Sullivan M, Dandapani V et al. (1993). Osteoblastic gene expression during adiposegenesis in hematopoietic supporting murine bone marrow stromal cells. Journal of Cellular Physiology, 154: 317-328.

12. Taichman RS, Reilly MJ \& Emerson SG (1996). Human osteoblasts support human progenitor cells in in vitro bone marrow cultures. Blood, 87: 518-524.

13. Krebsbach P, Kuznetsov S, Satourma K et al. (1997). Bone formation in vivo: comparison of osteogenesis by transplanted mouse and human marrow stromal fibroblasts. Transplantation, 63: 1059-1069.

14. Sutherland HJ, Lansdorp PM, Henkelman DH et al. (1990). Functional characterization of individual human hematopoietic stem cells cultured at limiting dilution on supportive marrow stromal layers. Proceedings of the National Academy of Sciences, USA, 87: 35843588.

15. Taichman RS, Reilly MJ, Verma RS et al. (2001). Hepatocyte growth factor is secreted by osteoblasts and cooperatively permits the survival of haematopoietic progenitors. British Journal of Haematology, 112: 438-448.

16. Taichman RS \& Emerson SG (1998). The role of osteoblasts in the hematopoietic microenvironment. Stem Cells, 16: 7-15.

17. El-Badri NS, Wang BY, Cherry et al. (1998). Osteoblasts promote engraftment of allogeneic hematopoietic stem cells. Experimental
Hematology, 26: 110-116.

18. Taichman RS, Reilly MJ \& Matthews LS (2000). Human osteoblastlike cells and osteosarcoma cell lines synthesize MIP-1 $\alpha$ in response to IL-1B and TNF- $\alpha$ stimulation in vitro. British Journal of Haematology, 108: 275-283.

19. Taichman RS, Cooper C, Keller ET et al. (2002). Use of the stromal cell-derived factor-1/CXCR4 pathway in prostate cancer metastasis to bone. Cancer Research, 62: 1832-1837.

20. Zhang JW, Niu C, Ye L et al. (2003). Identification of the haematopoietic stem cell niche and control of the niche size. Nature, 425: 836-841.

21. Calvi LM, Adams GB, Weibrecht KW et al. (2003). Osteoblastic cells regulate the haematopoietic stem cell niche. Nature, 425: 841-846.

22. Arai F, Hirao A, Ohmura M et al. (2004). Tie2/angiopoietin-1 signaling regulates hematopoietic stem cell quiescence in the bone marrow niche. Cell, 118: 149-161.

23. Lemischka IR \& Moore KA (2003). Stem cells: interactive niches [comment]. Nature, 425: 778-779.

24. Puri MC \& Bernstein A (2003). Requirement for the TIE family of receptor tyrosine kinases in adult but not fetal hematopoiesis. Proceedings of the National Academy of Sciences, USA, 100: 1275312758.

25. Cahill RA, Jones C, Mueller TO et al. (2002). Replacement of recipient stromal/mesenchymal cells after bone marrow transplant using bone fragments and cultured osteoblast like cells. Blood, 100: 63 (Abstract).

26. Cahill RA, Klemperer MR, Steele A et al. (2001). Successful transplantation to correct a metabolic bone disease (infantile hypophosphatasia) using bone fragments plus cultured osteoblasts with T-cell depleted mismatched bone marrow lymphohematopoietic engraftment. Blood, 98: 796A-797A.

27. Skjodt H, Moller T \& Freiesleben SF (1989). Human osteoblast-like cells expressing $\mathrm{MHC}$ class-II determinants stimulate allogeneic and autologous peripheral-blood mononuclear-cells and function as antigen-presenting cells. Immunology, 68: 416-420.

28. Reyes-Botella CMM (2002). Antigenic phenotype of cultured human osteoblast-like cells. Cellular Physiology and Biochemistry, 12: 359364.

29. Imai K, Kobayashi M, Wang J et al. (1999). Selective transendothelial migration of hematopoietic progenitor cells: a role in homing of progenitor cells. Blood, 93: 149-156.

30. Lataillade JJ, Clay D, Dupuy C et al. (2000). Chemokine SDF-1 enhances circulating CD34+ cell proliferation in synergy with cytokines: possible role in progenitor survival. Blood, 95: 756-768.

31. Lataillade JJ, Clay D, Bourin P et al. (2002). Stromal cell-derived factor 1 regulates primitive hematopoiesis by suppressing apoptosis and by promoting G0/G1 transition in CD34+ cells: evidence for an autocrine/paracrine mechanism. Blood, 99: 1117-1129.

32. Aiuti A, Tavian M, Cipponi A et al. (1999). Expression of CXCR4, the receptor for stromal cell-derived factor-1 on fetal and adult human lympho-hematopoietic progenitors. European Journal of Immunology, 29: 1823-1831.

33. Nagasawa T, Hirota S, Tachibana K et al. (1996). Defects of B-cell lymphopoiesis and bone-marrow myelopoiesis in mice lacking the CXC chemokine PBSF/SDF-1. Nature, 382: 635-638.

34. Peled A, Petit I, Kollet O et al. (1999). Dependence of human stem cell engraftment and repopulation of NOD/SCID mice on CXCR4. Science, 283: 845-848. 
35. McGrath KE, Koniski AD, Maltby KM et al. (1999). Embryonic expression and function of the chemokine SDF-1 and its receptor, CXCR4. Developmental Biology, 213: 442-456.

36. Hamada T, Mohle R, Hesselgesser J et al. (1998). Transendothelial migration of megakaryocytes in response to stromal cell-derived factor 1 (SDF-1) enhances platelet formation. Journal of Experimental Medicine, 188: 539-548.

37. Wang JF, Liu ZY \& Groopman JE (1998). The alpha-chemokine receptor CXCR4 is expressed on the megakaryocytic lineage from progenitor to platelets and modulates migration and adhesion. Blood, 92: 756-764.

38. Yu XF, Huang YF, Collin-Osdoby $P$ et al. (2003). Stromal cellderived factor-1 (SDF-1) recruits osteoclast precursors by inducing chemotaxis, matrix metalloproteinase-9 (MMP-9) activity, and collagen transmigration. Journal of Bone and Mineral Research, 18: 1404-1418.

39. Yu XF, Collin-Osdoby $P$ \& Osdoby $P$ (2003). SDF-1 increases recruitment of osteoclast precursors by upregulation of matrix metalloproteinase-9 activity. Connective Tissue Research, 44: 79-84.

40. Petit I, Szyper-Kravitz M, Nagler A et al. (2002). G-CSF induces stem cell mobilization by decreasing bone marrow SDF-1 and upregulating CXCR4. Nature Immunology, 3: 687-694.

41. Rempel SA, Dudas S, Ge S et al. (2000). Identification and localization of the cytokine SDF1 and its receptor, CXC chemokine receptor 4 , to regions of necrosis and angiogenesis in human glioblastoma. Clinical Cancer Research, 6: 102-111.

42. Salvucci O, Yao L, Villalba S et al. (2002). Regulation of endothelial cell branching morphogenesis by endogenous chemokine stromalderived factor-1. Blood, 99: 2703-2711.

43. Sun Y-X, Wang J, Shelburne CE et al. (2003). The expression of CXCR4 and CXCL12 (SDF-1) in human prostate cancers (PCa) in vivo. Journal of Cellular Biochemistry, 89: 462-473.

44. Kang YB, Siegel PM, Shu WP et al. (2003). A multigenic program mediating breast cancer metastasis to bone. Cancer Cells, 3: 537549.

45. Sun $Y X$, Schneider A, Jung $Y$ et al. (2005). Skeletal localization and neutralization of the SDF-1(CXCL12)/CXCR4 axis blocks prostate cancer metastasis and growth in osseous sites in vivo. Journal of Bone and Mineral Research, 20: 318-329. 